\title{
Incidence and Clinical Significance of Acute Reocclusion after Emergent Angioplasty or Stenting for Underlying Intracranial Stenosis in Patients with Acute Stroke
}

\author{
(D) G.E. Kim, DW. Yoon, (1) S.K. Kim, DB.C. Kim, DT.W. Heo, DB.H. Baek, (D)Y.Y. Lee, and DN.Y. Yim
}

\begin{abstract}
BACKGROUND AND PURPOSE: A major concern after emergent intracranial angioplasty in cases of acute stroke with underlying intracranial stenosis is the acute reocclusion of the treated arteries. This study reports the incidence and clinical outcomes of acute reocclusion of arteries following emergent intracranial angioplasty with or without stent placement for the management of patients with acute stroke with underlying intracranial atherosclerotic stenosis.
\end{abstract}

MATERIALS AND METHODS: Forty-six patients with acute stroke received emergent intracranial angioplasty with or without stent placement for intracranial atherosclerotic stenosis and underwent follow-up head CTA. Acute reocclusion was defined as "hypoattenuation" within an arterial segment with discrete discontinuation of the arterial contrast column, both proximal and distal to the hypoattenuated lesion, on CTA performed before discharge. Angioplasty was defined as "suboptimal" if a residual stenosis of $\geq 50 \%$ was detected on the postprocedural angiography. Clinical and radiologic data of patients with and without reocclusion were compared.

RESULTS: Of the 46 patients, 29 and 17 underwent angioplasty with and without stent placement, respectively. Acute reocclusion was observed in 6 patients (13\%) and was more frequent among those with suboptimal angioplasty than among those without it (71.4\% versus $2.6 \%, P<.001)$. The relative risk of acute reocclusion in patients with suboptimal angioplasty was 27.857 ( $95 \%$ confidence interval, 3.806-203.911). Furthermore, a good outcome was significantly less frequent in patients with acute reocclusion than in those without it (16.7\% versus $67.5 \%, P=.028)$.

CONCLUSIONS: Acute reocclusion of treated arteries was common after emergent intracranial angioplasty with or without stent placement in patients with acute stroke with intracranial atherosclerotic stenosis and was associated with a poor outcome. Suboptimal results of angioplasty appear to be associated with acute reocclusion, irrespective of whether stent placement was performed.

ABBREVIATIONS: ICAS = intracranial atherosclerotic stenosis; IQR $=$ interquartile range

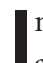

ntracranial atherosclerotic stenosis (ICAS) is rapidly becoming a major concern worldwide in patients with stroke because Asian, Hispanic, and African populations, which are prone to ICAS, constitute an overwhelming majority of the population of the world. ${ }^{1}$ Acute stroke caused by an in situ thrombosis at the site of ICAS differs from that caused by cardiogenic embolic occlusion and may not respond to modern mechanical embolectomy procedures such as stent-retriever embolectomy or manual aspiration embolectomy. Few studies have been published hitherto on

Received November 18, 2015; accepted after revision February 9, 2016 From the Departments of Radiology (G.E.K., W.Y., S.K.K., T.W.H., B.H.B., Y.Y.L., N.Y.Y.) and Neurology (B.C.K.), Chonnam National University Medical School, Chonnam National University Hospital, Gwangju, Republic of Korea.

Please address correspondence to Woong Yoon, MD, Department of Radiology, Chonnam National University Hospital, 42 Jebong-ro, Dong-gu, Gwangju, 501-757, Republic of Korea; e-mail: radyoon@jnu.ac.kr

http://dx.doi.org/10.3174/ajnr.A4770 the optimal treatment plan for patients with acute stroke with underlying ICAS. ${ }^{2-5}$ Emergent intracranial angioplasty, with or without stent placement, has been recently suggested as an effective treatment option for the management of underlying ICAS following mechanical embolectomy; for example, Yoon et $\mathrm{al}^{4} \mathrm{re-}$ ported a successful revascularization rate of $95 \%$ and a favorable outcome rate of $65 \%$ with this strategy.

Nevertheless, a major concern after emergent intracranial angioplasty in cases of acute stroke is the acute reocclusion of the treated arteries. Acute reocclusion may occur following intracranial angioplasty or stent placement as a result of platelet activation, adherence, and aggregation triggered by the disruption of vulnerable plaque and endothelial damage; unsuccessful or inadequate pretreatment with antiplatelet drugs; dissection; or vasospasm. ${ }^{6}$ Despite the possibility of this complication in patients with acute stroke, data regarding its incidence and clinical consequences have not yet been reported in literature, to our knowledge. 
Thus, this study aimed to investigate the incidence and clinical outcomes of arterial reocclusion in patients with acute stroke undergoing emergent angioplasty with or without stent placement following stent-retriever embolectomy. Additionally, we sought to determine the risk factors associated with acute reocclusion in these patients.

\section{MATERIALS AND METHODS Patients}

Between January 2011 and February 2014, 240 patients with acute ischemic stroke were treated with mechanical embolectomy at our comprehensive regional stroke center. Of these, 50 (20.8\%) consecutive patients with underlying ICAS as a cause of acute large-vessel occlusion underwent emergent intracranial angioplasty with or without stent placement subsequent to mechanical embolectomy. Forty-six of these patients underwent follow-up head CTA after endovascular therapy, as per our institutional protocol for acute stroke management. The remaining 4 patients could not undergo CTA during hospitalization due to their poor health status. Thus, 46 patients were finally enrolled in this retrospective study, and their clinical and radiologic data were analyzed. The institutional ethics committee approved this retrospective analysis and waived informed consent due to the retrospective study design.

On admission, a stroke neurologist performed an assessment by using the NIHSS. Before the initiation of endovascular treatment, all patients underwent nonenhanced cranial CT and multimodal MR imaging. The inclusion criteria for endovascular therapy were as follows: presentation within 6 and 8 hours of stroke onset for anterior and posterior circulation stroke, respectively; a baseline NIHSS score of $\geq 4$; no evidence of intracranial hemorrhage on cranial CT or MR imaging; evidence of major arterial occlusion on MR angiography; a target mismatch pattern on multimodal MR imaging based on visual estimation (time-topeak map of perfusion imaging showing a lesion volume $\geq 30 \%$ larger than that detected with DWI) for an anterior circulation stroke; an infarct volume of less than one-third of the MCA territory for anterior circulation stroke, as determined by DWI or nonenhanced CT; an absence of bilateral diffuse pontine ischemia on DWI for posterior circulation stroke; and a premorbid mRS score of $\leq 3$.

\section{Endovascular Therapy}

All endovascular therapy was performed by a single interventional neuroradiologist with 12 years of experience in neurovascular intervention. For each patient, written informed consent for endovascular therapy was obtained from a family member. Both cerebral angiography and endovascular therapy were performed with the patient under conscious sedation. In case of agitation, an intravenous bolus of midazolam was administered and repeated if necessary. When pretreatment diagnostic angiography showed an arterial occlusion, stent-retriever embolectomy with the Solitaire stent (Covidien, Irvine, California) was performed as the first-line endovascular treatment. When stent-retriever embolectomy was unsuccessful, other mechanical approaches were performed, including manual aspiration embolectomy by using a Penumbra aspiration catheter (Penumbra, Alameda, California). The details of the techniques used for mechanical embolectomy have been described previously. ${ }^{7,8}$

If severe ( $>70 \%)$ underlying ICAS of the target artery was seen on the initial diagnostic angiography or on follow-up angiography after mechanical embolectomy, intracranial angioplasty with or without stent placement was performed. The severity of arterial stenosis was graded according to the Warfarin-Aspirin Symptomatic Intracranial Disease criteria. ${ }^{9}$ For underlying ICAS seen after the initial mechanical embolectomy, ICAS was determined if the stenotic lesion remained unchanged 3-5 minutes after intra-arterial injection of a vasodilator through the guide catheter. The devices and techniques used for intracranial angioplasty and stent placement have been described in detail elsewhere. ${ }^{4}$

Neither heparin nor glycoprotein IIb/IIIa inhibitor was administered either intravenously or intra-arterially during the endovascular procedure. All patients underwent nonenhanced CT immediately after endovascular therapy. If no intracranial hemorrhage was detected on this scan, aspirin and clopidogrel (Plavix) were administered orally via the nasogastric tube after the procedure. Dual antiplatelet therapy was then continued for at least 3 months after the procedure.

\section{CTA Image Acquisition and Analysis}

CTA was performed on a dual-source CT scanner (Somatom Definition Flash; Siemens, Erlangen, Germany) with the following parameters: collimation, $128 \times 0.6 \mathrm{~mm}$; tube voltage, $120 \mathrm{kV}$; tube current, $250 \mathrm{~mA}$; gantry rotation, 0.28 seconds; pitch factor, 0.6 ; FOV, $180 \mathrm{~mm}$; and matrix, $512 \times 512$. All patients were administered $100 \mathrm{~mL}$ of nonionic contrast agent with $350 \mathrm{mg}$ of iodine per milliliter (iohexol, Omnipaque 350; GE Healthcare, Piscataway, New Jersey), which was injected into the antecubital vein at a rate of $4 \mathrm{~mL} / \mathrm{s}$. Scanning was triggered by the bolus-tracking technique with the ROI positioned in the aortic arch and the trigger threshold set at $25 \mathrm{HU}$. CTA source images were then reconstructed with a section width of $0.75 \mathrm{~mm}$ for further evaluation. The source images were transferred to the workstation and processed with the use of a commercial software package (syngo.CT Dynamic Angio; Siemens). Thick, maximum-intensity-projection images (section thickness, $30 \mathrm{~mm}$; increments, $5 \mathrm{~mm}$ ) were reconstructed in axial, coronal, sagittal, and oblique coronal planes to evaluate the intracranial arteries.

Maximum-intensity-projection and source images of all patients were reviewed by 2 neuroradiologists and were evaluated for the presence or absence of occlusion or stenosis of $\geq 50 \%$ at the site of previous angioplasty or stent placement. Decisions were made by consensus between the 2 investigators. Arterial occlusion was defined as a hypoattenuation within the arterial segment with discrete discontinuation of the arterial contrast column between the proximal and distal points of the hypoattenuated lesion.

\section{Outcome Measures}

We recorded the clinical and radiologic data from the medical records of the patients. The "start of endovascular therapy" was defined as the moment the needle punctured the common femoral artery. "Symptomatic intracranial hemorrhage" was defined as any intracranial hemorrhage that caused neurologic deteriora- 


\begin{tabular}{|c|c|c|c|c|}
\hline & $\begin{array}{l}\text { All Patients } \\
\qquad(N=46)\end{array}$ & $\begin{array}{l}\text { Patients with } \\
\text { Acute Reocclusion } \\
\qquad(n=6)\end{array}$ & $\begin{array}{l}\text { Patients without } \\
\text { Acute Reocclusion } \\
\qquad(n=40)\end{array}$ & $P$ Value \\
\hline Age (yr) (IQR) & $66(57.75-74.75)$ & $63(57.5-72.5)$ & $66(57.5-76.25)$ & NS \\
\hline Male sex (No.) (\%) & $28(60.9)$ & $3(50)$ & $25(62.5)$ & NS \\
\hline \multicolumn{5}{|l|}{ Risk factors (No.) (\%) } \\
\hline Hypertension & $29(63)$ & $4(66.7)$ & $25(62.5)$ & NS \\
\hline Diabetes mellitus & $20(43.6)$ & $2(33.3)$ & $18(45)$ & NS \\
\hline Dyslipidemia & $26(56.5)$ & $4(66.7)$ & $22(55)$ & NS \\
\hline Smoking & $10(21.7)$ & $2(33.3)$ & $8(20)$ & NS \\
\hline Atrial fibrillation & $4(8.7)$ & $0(0)$ & $4(10)$ & NS \\
\hline Previous CAD & $1(2.2)$ & $0(0)$ & $1(2.5)$ & NS \\
\hline Congestive heart failure & $1(2.2)$ & $0(0)$ & $1(2.5)$ & NS \\
\hline History of stroke or TIA & $8(17.4)$ & $4(66.7)$ & $4(10)$ & .005 \\
\hline \multicolumn{5}{|l|}{ Occlusion site (No.) (\%) } \\
\hline Middle cerebral artery & $26(56.5)$ & $5(83.3)$ & $21(52.5)$ & NS \\
\hline Internal carotid artery & $8(17.4)$ & $0(0)$ & $8(20)$ & NS \\
\hline Basilar artery & $12(26.1)$ & $1(16.7)$ & $11(27.5)$ & NS \\
\hline Baseline NIHSS score (IQR) & $12(8-14)$ & $9.5(6.5-14.5)$ & $10(9-14)$ & NS \\
\hline Intravenous thrombolysis (No.) (\%) & $18(39.1)$ & $4(66.7)$ & $14(35)$ & NS \\
\hline Intra-arterial thrombolysis (No.) (\%) & $7(15.2)$ & $1(16.7)$ & $6(15)$ & NS \\
\hline Time to procedure (min) (IQR) & $270(207.75-362)$ & $277.5(233.75-330.00)$ & $252.5(187.5-360)$ & NS \\
\hline Procedural time (min) (IQR) & $36(25-46.75)$ & $33(22.75-47.25)$ & $37(25.75-48.75)$ & NS \\
\hline Time to revascularization (min) (IQR) & $319.5(231.75-401.5)$ & $305(264.75-385.75)$ & $301(217.5-407)$ & NS \\
\hline Postprocedural residual stenosis (\%) (IQR) & $25(19.5-35.0)$ & $56(42.3-71.3)$ & $25(19-33)$ & 0.009 \\
\hline Postprocedural antiplatelet therapy (No.) (\%) & $41(89.1)$ & $6(100)$ & $35(87.5)$ & NS \\
\hline
\end{tabular}

Note:-CAD indicates coronary artery disease; NS, not significant.

tion, which was a $\geq 4$-point increase in the NIHSS score or a 1 -point deterioration in the level of consciousness.

Revascularization status was assessed by using the final angiogram according to the modified TICI scale, ${ }^{10}$ with "successful revascularization" defined as a modified TICI grade of $2 \mathrm{~b}$ or 3 . "Arterial perforation" was defined by evidence of frank angiographic contrast extravasation on serial angiograms. "Arterial dissection" was defined as an identifiable, intimal flap on the final angiogram. The angiographic findings were characterized as either "optimal" ( $<50 \%$ residual stenosis) or "suboptimal" ( $\geq 50 \%$ residual stenosis) on the final angiogram. ${ }^{11,12}$ Angiographic images were evaluated by 2 neuroradiologists in consensus.

Neurologic evaluation was performed by a stroke neurologist immediately and 24 hours after treatment, before discharge, 3 months after treatment, and when there were any changes in clinical symptoms. Clinical outcome was assessed by a stroke neurologist by using the mRS score during an outpatient visit 3 months after treatment. If patients were unable to attend the outpatient clinic, assessment was made via telephone interviews. A good clinical outcome was defined as an mRS score of $\leq 2$ or equal to the premorbid mRS score.

\section{Statistical Analysis}

Continuous variables are presented as medians and interquartile ranges (IQRs). Discrete variables are presented as counts and percentages. First, patients were divided into 2 groups for comparison, according to the presence or absence of acute reocclusion on head CTA. The baseline characteristics and clinical outcomes of the 2 groups were compared. Second, the relationship between each clinical and radiologic characteristic and the 3-month outcome was determined. The $\chi^{2}$ or Fisher exact test was used for comparing categoric and binary data, and the Mann-Whitney $U$ test was used for comparing continuous data. Third, a logistic regression analysis was performed to identify independent predictors of good clinical outcome. The variables tested in the logistic regression models were those with $P<.05$ in the univariate analysis, age, and successful revascularization. All statistical analyses were performed with SPSS software (Version 21.0; IBM, Armonk, New York). A $P$ value $<.05$ was significant.

\section{RESULTS}

Data from 46 patients were analyzed. Baseline patient characteristics are shown in Table 1. Of the 46 patients treated with emergent angioplasty with or without stent placement for acute stroke, 26 had ICAS in the MCA; 12, in the basilar artery; and 8 , in the intracranial internal carotid artery. Follow-up CTA was performed within 48 hours of endovascular therapy in 45 patients $(97.8 \%)$. The remaining patient underwent CTA 120 hours after endovascular therapy.

Follow-up CTA showed reocclusion at the site of angioplasty or stent placement in 6 patients (13\%), with reocclusion occurring in the MCA in 5 patients and in the basilar artery in 1 patient (Fig 1). A history of previous stroke or TIA was more frequent in patients with reocclusion $(66.7 \%$ versus $10 \%, P=.005)$ than in those without it. Postprocedural residual stenosis was significantly greater in patients with acute reocclusion (56\% versus $25 \%$, $P=.009)$. No significant intergroup differences were noted in terms of patient age, sex, history of other risk factors, occlusion sites, use of intravenous or intra-arterial thrombolysis, time to procedure, duration of the procedure, time to revascularization, and baseline NIHSS score. Severe stenosis $(\geq 50 \%)$ at the site of angioplasty or stent placement was not observed in the remaining 40 patients.

Thirty-two patients underwent both intracranial angioplasty and stent placement, while 14 patients underwent only angio- 

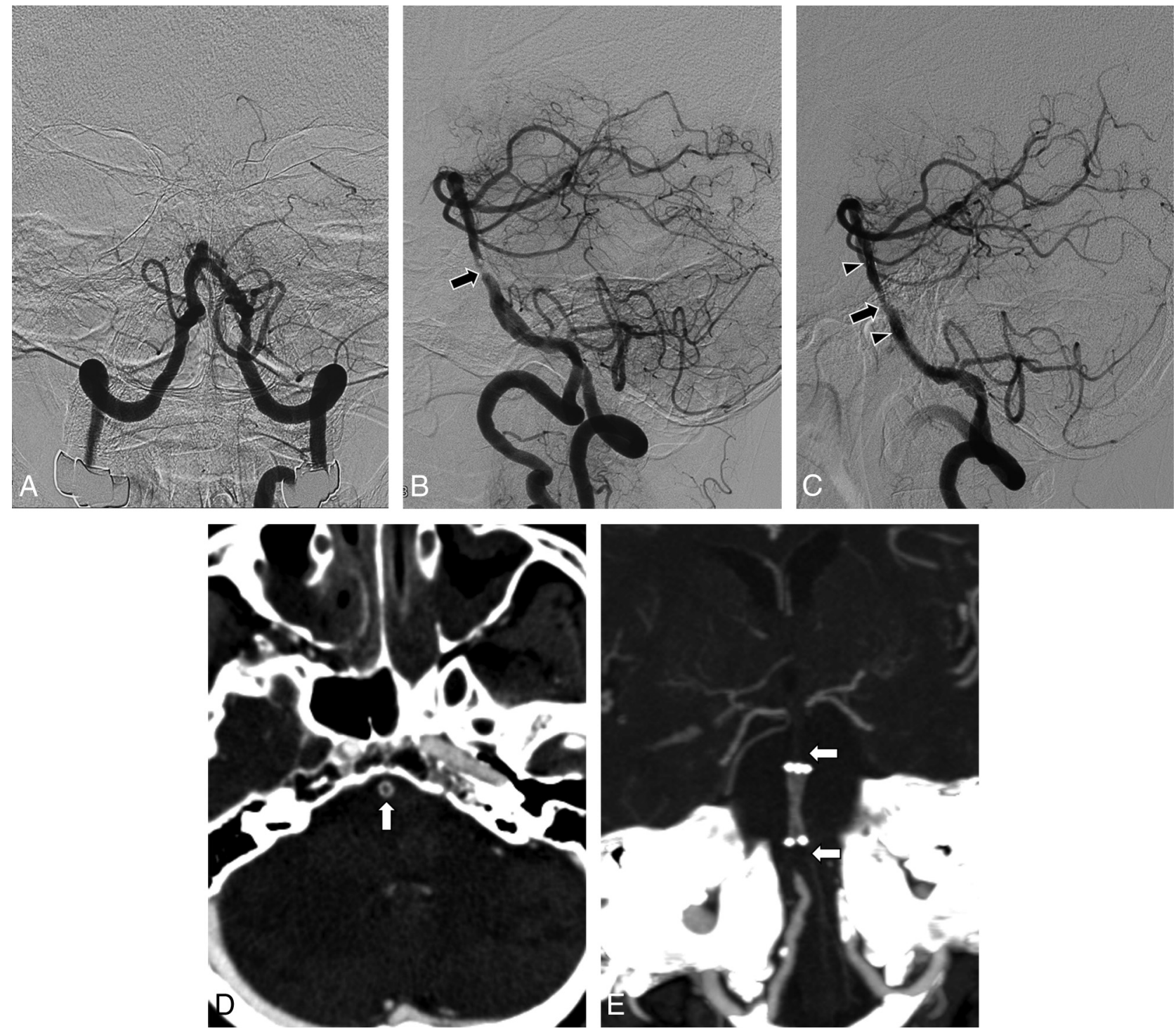

FIG 1. Brain images obtained in a patient with acute stroke. A, Anteroposterior view of a vertebral angiogram shows arterial occlusion at the proximal portion of the basilar artery. $B$, Lateral projection of the vertebral angiogram obtained after stent-retriever embolectomy reveals severe underlying stenosis (arrow) at the site of arterial occlusion. There were no retrieved thrombi with the Solitaire stent. C, Lateral projection of vertebral angiography performed after intracranial stent placement shows suboptimal angioplasty, with residual stenosis of $60 \%$ (arrow). Arrowheads indicate the proximal and distal ends of the stent. D, Axial source image of CTA shows hypoattenuation (arrow) within the stented segment of the basilar artery. E, Maximum-intensity-projection image of the follow-up CTA 2 days after the procedure shows a discontinuation of the contrast column both proximal and distal to the stent (arrows).

plasty. Acute reocclusion was more frequent among patients treated with intracranial angioplasty alone than among those treated with intracranial stent placement in addition to angioplasty (23.5\% versus 6.9\%); however, this difference was not statistically significant $(P=.174)$. Of the 46 patients, 41 received antiplatelet medication after completion of endovascular therapy; 5 patients did not receive this therapy because they showed evidence of intracranial hemorrhage in the postprocedural CT scan. However, none of these 5 patients exhibited acute reocclusion on follow-up CTA.

Of 6 patients with reocclusion, 4 patients had an ASPECTS of 8, 1 had an ASPECTS of 7, and 1 had a posterior circulation ASPECTS of 5 on pretreatment DWI. The NIHSS score gradually increased by $2-5$ points during the first 24 hours after endovascular therapy in all 6 patients with reocclusion. At the time of dis- charge, the NIHSS score was unchanged in 3 patients, decreased in 2 patients (by 2 and 4 points, respectively), and slightly increased in 1 patient (by 3 points) compared with the score on day 1.

The clinical outcomes of patients with or without acute reocclusion are shown in Table 2. Overall, successful revascularization (modified TICI grade $2 \mathrm{~b}$ or 3 ) was achieved in 95.7\% (44/46) of patients. There was no significant difference between the 2 groups in terms of the rate of successful revascularization. A suboptimal result ( $\geq 50 \%$ residual stenosis at the site of angioplasty or stent placement) was observed on the final angiograms in 7 patients (15.2\%). Furthermore, acute reocclusion was more frequent among patients with a suboptimal result than among those without $(71.4 \%$ [5/7] versus $2.6 \%$ [1/39], $P<.001)$. The relative risk for acute reocclusion in patients with a suboptimal result was 
Table 2: Comparison of clinical outcomes between patients with and without acute reocclusion

\begin{tabular}{lcccc}
\hline & $\begin{array}{c}\text { All } \\
\text { Patients } \\
(\mathbf{N}=\mathbf{4 6})\end{array}$ & $\begin{array}{c}\text { Patients } \\
\text { with Acute } \\
\text { Reocclusion } \\
(\boldsymbol{n}=6)\end{array}$ & $\begin{array}{c}\text { Patients } \\
\text { without Acute } \\
\text { Reocclusion } \\
(\boldsymbol{n}=\mathbf{4 0 )}\end{array}$ & $\boldsymbol{P}$ Value \\
\hline m-TICl grade 2b or 3 (No.) (\%) & $44(95.7)$ & $5(83.3)$ & $39(97.5)$ & NS \\
Suboptimal result (No.) (\%) & $7(15.2)$ & $5(83.3)$ & $2(5)$ & $<.001$ \\
mRS 0-2 (No.) (\%) & $28(60.9)$ & $1(16.7)$ & $27(67.5)$ & .028 \\
Symptomatic hemorrhage (No.) (\%) & $2(4.3)$ & $0(0)$ & $2(5)$ & NS \\
Mortality (No.) (\%) & $3(6.5)$ & $0(0)$ & $3(7.5)$ & NS \\
\hline
\end{tabular}

Note:-m-TICl indicates modified TICl; NS, not significant.

27.857 (95\% CI, 3.806-203.911). Overall, a good clinical outcome was achieved in $60.9 \%$ (28/46) of patients. A good outcome was less frequent in patients with acute reocclusion than in those without it $(16.7 \%$ versus $67.5 \%, P=.028)$; the relative risk of a poor outcome in patients with acute reocclusion was $2.564(95 \%$ CI, 1.447-4.544).

Arterial perforation of a thalamoperforating artery by microguidewire occurred in 1 patient. Arterial dissection was not observed in the postprocedural angiograms of any patients. Overall, 2 patients $(4.3 \%)$ without acute reocclusion had symptomatic hemorrhage, while 3 patients $(6.5 \%)$ without acute reocclusion died before the 3-month follow-up. However, none of the patients with acute reocclusion showed symptomatic hemorrhage or 3-month mortality. There was no significant difference between the 2 groups in the rate of symptomatic hemorrhage and mortality.

In a univariate analysis, the following variables were identified as predictors of a good outcome at 3 months: acute reocclusion, history of stroke or TIA, and procedural time. A good outcome occurred less frequently among patients with a history of previous stroke or TIA than among those without $(12.5 \%$ [1/8] versus $71.1 \%$ [27/38], $P=.004)$. The median procedural time was shorter in patients with a good outcome than in those with a poor outcome (35 minutes [IQR, 25-42 minutes] versus 44 minutes [IQR, 32.75-62 minutes], $P=.019)$. When acute reocclusion, history of previous stroke or TIA, procedural time, each year of age, and successful revascularization were selected for the logistic regression analysis, independent predictors of good outcome were a history of previous stroke or TIA (OR, 0.055; 95\% CI, $0.004-0.821 ; P=.035)$ and procedural time (per 1-minute decrease; OR, 1.046; 95\% CI, 1.000-1.093; $P=.048)$.

\section{DISCUSSION}

The main findings of this study are summarized as follows: 1) acute reocclusion of arteries treated with emergent angioplasty with or without stent placement was fairly common in this study population, at a rate of $13 \%$; 2) suboptimal angioplasty with or without stent placement was significantly associated with acute reocclusion; and 3) acute reocclusion was significantly associated with a poor outcome at 3-month follow-up.

Few studies have been published thus far on the incidence of acute reocclusion after intracranial angioplasty in acute stroke. Consistent with our study, Lee et $\mathrm{al}^{2}$ reported that early reocclusion occurred within 48 hours in $16.7 \%$ (2/12) of patients with acute MCA occlusion after intracranial stent placement for the treatment of underlying ICAS. Additionally, Guo et $\mathrm{al}^{13}$ reported that acute reocclusion occurred in $9.1 \%(1 / 11)$ of patients within
1 day of intracranial stent placement. Moreover, Bang et $\mathrm{al}^{14}$ reported that instent thrombosis occurred within 1 week in $18.8 \%(6 / 32)$ of their patients who underwent intracranial stent placement for acute ischemic stroke. However, the latter 2 studies differ from our study and that of Lee et al in that stent placement was attempted to recanalize the embolic occlusions and not to treat the underlying ICAS. Furthermore, those studies did not provide data on specific stroke etiology or postprocedural antiplatelet medication for the treated patients.

In our study, suboptimal angioplasty ( $\geq 50 \%$ residual stenosis) seemed to be a main predisposing factor for acute reocclusion. Suboptimal angioplasty was associated with a 28 -fold increase in the rate of acute reocclusion in the present study. A suboptimal result may cause insufficient blood flow through the angioplastytreated or stented arterial segment, thereby accelerating platelet aggregation. Sufficient blood flow at the site of angioplasty, with or without stent placement, prevents acute thrombus formation. ${ }^{15}$ Thus, our findings strongly suggest that neurointerventionists should pay particular attention to achieving optimal patency of the target artery during emergent intracranial angioplasty or stent placement for the treatment of underlying ICAS in patients with acute stroke.

A systematic review has shown that the frequency of reocclusion after stent placement is lower than after angioplasty alone in patients undergoing acute coronary interventions. ${ }^{16}$ Similarly, intracranial stent placement has been shown to be more effective than angioplasty alone in postoperative residual stenosis or late restenosis in cases of subacute-to-chronic symptomatic ICAS. ${ }^{12,17}$ Stent placement has been associated with a lower incidence of acute reocclusion than angioplasty alone, by eliminating vascular recoil and dissection and allowing increased vessel diameter. ${ }^{15}$ In our study, we also noted a trend toward a lower incidence of acute reocclusion among patients treated with stent placement than among those treated with angioplasty alone (6.9\% versus $23.5 \%)$; however, this difference was not statistically significant, probably due to the small sample size.

Angioplasty with or without stent placement may lead to mechanical disruption of atherosclerotic plaques or endothelial injury that triggers platelet activation, adhesion, and aggregation, which are key features of arterial thrombosis and reocclusion. Therefore, adequate antiplatelet therapy is generally recommended to prevent acute thrombosis and reocclusion in cases of scheduled intracranial angioplasty or stent placement. Although optimal doses of antiplatelet therapy for periprocedural support during endovascular stroke therapy have not yet been established, loading doses of aspirin (300-650 mg) and clopidogrel (600 mg) are generally recommended, followed by daily doses of aspirin (100-300 mg) and clopidogrel (75 mg) for $1-3$ months. ${ }^{18}$ Most interesting, the administration of periprocedural antiplatelet therapy did not appear to be associated with acute reocclusion in our study. There was no acute reocclusion in the 5 patients who did not receive postprocedural antiplatelet therapy in our study. All patients with acute reocclusion received loading doses of aspirin and clopidogrel after the procedure. Therefore, further studies 
are necessary to determine the exact role of periprocedural antiplatelet therapy in patients undergoing emergency angioplasty with or without stent placement for the management of acute ischemic stroke in patients with ICAS.

There have been no studies to date on the clinical consequences of acute reocclusion after emergency angioplasty with or without stent placement in patients with acute stroke due to ICAS. In our study, a good outcome was considerably less frequent among patients with acute reocclusion than among those without it ( $16.7 \%$ versus $67.5 \%, P=.028$ ); in fact, acute reocclusion was associated with a 2.6-fold increase in poor outcome at the 3-month follow-up. Similar results have been reported in a study on the immediate reocclusion of recanalized arteries during intra-arterial thrombolysis for embolic occlusion. Janjua et $\mathrm{al}^{19}$ showed that a favorable outcome was less frequently achieved in patients with immediate arterial reocclusion after various endovascular treatments than in those without it (6\% versus $37 \%, P=.01$ ). The odds ratio of a poor outcome in patients with arterial reocclusion was 3.9 in their analysis. ${ }^{19}$

The limitations of our study include its small sample size and retrospective design. While most patients (97.8\%) underwent head CTA 2 days after endovascular therapy, 1 patient underwent the procedure 5 days after the operation. In addition, platelet function testing was not routinely performed. Thus, individualizing antiplatelet therapy was not possible; this situation may have influenced the incidence of acute reocclusion in this study. However, the impact of clopidogrel resistance on the effectiveness of endovascular therapy for acute ischemic stroke remains largely unstudied. Furthermore, routine platelet-function testing is not currently recommended in cases of acute coronary intervention. ${ }^{18,20}$

\section{CONCLUSIONS}

Our findings indicate that acute reocclusion of treated vessels with or without stent placement after emergent angioplasty is common in patients with acute stroke due to ICAS and that this complication is associated with a poor outcome. Acute reocclusion in such cases appears to be associated with suboptimal angioplasty or stent placement. In light of these results, neurointerventionists should make every effort to achieve optimal results in restoring the patency of the affected artery during emergent angioplasty in cases of acute stroke due to ICAS.

\section{REFERENCES}

1. Gorelick PB, Wong KS, Bae HJ, et al. Large artery intracranial occlusive disease: a large worldwide burden but a relatively neglected frontier. Stroke 2008;39:2396-99 CrossRef Medline

2. Lee HK, Kwak HS, Chung GH, et al. Balloon-expandable stent placement in patients with immediate reocclusion after initial successful thrombolysis of acute middle cerebral arterial obstruction. Interv Neuroradiol 2012;18:80-88 Medline

3. Kang DH, Kim YW, Hwang YH, et al. Instant reocclusion following mechanical thrombectomy of in situ thromboocclusion and the role of low-dose intra-arterial tirofiban. Cerebrovasc Dis 2014;37: 350-55 CrossRef Medline
4. Yoon W, Kim SK, Park MS, et al. Endovascular treatment and the outcomes of atherosclerotic intracranial stenosis in patients with hyperacute stroke. Neurosurgery 2015;76:680-86; discussion 686 CrossRef Medline

5. Behme D, Weber W, Mpotsaris A. Acute basilar artery occlusion with underlying high-grade basilar artery stenosis: multimodal endovascular therapy in a series of seven patients. Clin Neuroradiol 2015;25:267-74 CrossRef Medline

6. Qureshi AI, Siddiqui AM, Kim SH, et al. Reocclusion of recanalized arteries during intra-arterial thrombolysis for acute ischemic stroke. AJNR Am J Neuroradiol 2004;25:322-28 Medline

7. Yoon W, Jung MY, Jung SH, et al. Subarachnoid hemorrhage in a multimodal approach heavily weighted toward mechanical thrombectomy with Solitaire stent in acute stroke. Stroke 2013;44:414-19 CrossRef Medline

8. Kim SK, Yoon W, Moon SM, et al. Outcomes of manual aspiration thrombectomy for acute ischemic stroke refractory to stent-based thrombectomy. J Neurointerv Surg 2015;7:473-77 CrossRef Medline

9. Samuels OB, Joseph GJ, Lynn MJ, et al. A standardized method for measuring intracranial arterial stenosis. AJNR Am J Neuroradiol 2000;21:643-46 Medline

10. Zaidat OO, Yoo AJ, Khatri P, et al; Cerebral Angiographic Revascularization Grading (CARG) Collaborators, STIR Revascularization working group, STIR Thrombolysis in Cerebral Infarction (TICI) Task Force. Recommendations on angiographic revascularization grading standards for acute ischemic stroke: a consensus statement. Stroke 2013;44:2650-63 CrossRef Medline

11. Yoon W, Seo JJ, Cho KH, et al. Symptomatic middle cerebral artery stenosis treated with intracranial angioplasty: experience in $32 \mathrm{pa}-$ tients. Radiology 2005;237:620-26 CrossRef Medline

12. Siddiq F, Memon MZ, Vazquez G, et al. Comparison between primary angioplasty and stent placement for symptomatic intracranial atherosclerotic disease: meta-analysis of case series. Neurosurgery 2009;65:1024-33; discussion 1033-34 CrossRef Medline

13. Guo XB, Song LJ, Guan S. Emergent angioplasty and stent placement recanalization without thrombolysis in acute middle cerebral artery occlusions. J Stroke Cerebrovasc Dis 2013;22:694-99 CrossRef Medline

14. Bang JS, Oh CW, Jung C, et al. Intracranial stent placement for recanalization of acute cerebrovascular occlusion in 32 patients. AJNR Am J Neuroradiol 2010;31:1222-25 CrossRef Medline

15. Brekenfeld C1, Tinguely P, Schroth G, et al. Percutaneous transluminal angioplasty and stent placement in acute vessel occlusion: evaluation of new methods for interventional stroke treatment. AJNR Am J Neuroradiol 2009;30:1165-72 CrossRef Medline

16. Wilson SH, Bell MR, Rihal CS, et al. Infarct artery reocclusion after primary angioplasty, stent placement, and thrombolytic therapy for acute myocardial infarction. Am Heart J 2001;141:704-10 CrossRef Medline

17. Siddiq F, Vazquez G, Memon MZ, et al. Comparison of primary angioplasty with stent placement for treating symptomatic intracranial atherosclerotic diseases: a multicenter study. Stroke 2008;39: 2505-10 CrossRef Medline

18. Nahab F, Kass-Hout T, Shaltoni HM. Periprocedural antithrombotic strategies in acute ischemic stroke interventional therapy. Neurology 2012;79(13 suppl 1):S174-81 CrossRef Medline

19. Janjua N, Alkawi A, Suri MF, et al. Impact of arterial reocclusion and distal fragmentation during thrombolysis among patients with acute ischemic stroke. AJNR Am J Neuroradiol 2008;29:253-58 CrossRef Medline

20. Yousuf $\mathrm{O}$, Bhatt DL. The evolution of antiplatelet therapy in cardiovascular disease. Nat Rev Cardiol 2011;8:547-59 CrossRef Medline 